\title{
Retinopathy of prematurity in the United Kingdom: retreatment rates, visual and structural 1-year outcomes
}

\author{
Gillian GW Adams ${ }^{1}$ - Catey Bunce $\mathbb{1}^{1,2} \cdot$ Wen Xing $^{1} \cdot$ Lucilla Butler $^{3} \cdot$ Vernon Long $^{4} \cdot$ Aravind Reddy $^{5}$. \\ Annegret H. Dahlmann-Noor ${ }^{1}$. UK Retinopathy of Prematurity Special Interest Group
}

Received: 27 September 2017 / Accepted: 6 June 2018 / Published online: 16 July 2018

(c) The Royal College of Ophthalmologists 2018

\begin{abstract}
Aims To evaluate retreatment rates, visual and anatomical outcomes at 1-year postnatal age in infants treated for retinopathy of prematurity (ROP)

Methods Longitudinal national surveillance study of infants treated for ROP in the United Kingdom between December 2013 and December 2014, supported by the British Ophthalmic Surveillance Unit. Here we report retreatment rates, anatomical, visual and refractive outcomes at 1-year follow-up.

Results One-year follow-up forms were completed for 168 children of the original cohort of 327 (51.4\%). Twenty-two had at least one retreatment: 17/153 right eyes (REs, 11.1\%) after initial diode laser, and 5/14 REs (35.7\%) after initial injection of anti-vascular endothelial growth factor (VEGF) antibody. Median (interquartile range) RE best-corrected visual acuity was $0.6(0.4-1.0)(n=46 \mathrm{REs})$, and median acuity both eyes open $0.4(0.3-0.7) \operatorname{logMAR}(n=89)$. Median spherical equivalent (RE) was $0.44(-1.3$ to 1.3$)$ dioptre (D) $(n=116)$. Median astigmatism (RE) was $0.5(0-1.0) \mathrm{D}(n=111)$, and median anisometropia $0.125(0-0.75) \mathrm{D}(n=116)$. Twenty-four children $(20.5 \%)$ had been prescribed glasses. Sight impairment certification eligibility information was available for 131 children: $11(8.4 \%)$ were eligible to be certified as sight impaired, and 5 (3.8\%) as severely sight impaired.

Conclusions Retreatment rates are in line with previous reports, and appear higher after initial anti-VEGF antibody than after initial diode laser. Refractive outcomes are in line with previous studies, with a trend towards early emmetropia and myopia following diode laser, particularly in more severe ROP.
\end{abstract}

Members of the UK Retinopathy of Prematurity Special Interest Group are listed before the references.

Electronic supplementary material The online version of this article (https://doi.org/10.1038/s41433-018-0151-y) contains supplementary material, which is available to authorized users.

$\triangle$ Annegret H. Dahlmann-Noor

annegret.dahlmann-noor@moorfields.nhs.uk

$1 \quad$ NIHR Biomedical Research Centre at Moorfields Eye Hospital and UCL Institute of Ophthalmology, 162 City Road, London EC1V 2PD, UK

2 Department of Primary Care and Public Health Sciences, Kings College London, London SE1 1UL, UK

3 Birmingham and Midland Eye Centre, Dudley Road, Birmingham B18 7QH, UK

4 St James' University Hospital Leeds, Teaching Hospitals, Beckett Street, Leeds, West Yorkshire LS9 7TF, UK

5 Royal Aberdeen Children's Hospital, Westburn Road, Foresterhill, Aberdeen AB25 2ZG, UK

\section{Background}

Timely treatment for sight-threatening retinopathy of prematurity (ROP) aims to enable the child to develop normal vision. Ideally, one treatment application should induce permanent regression of ROP. Current standard treatment is diode laser ablation of the avascular retina, with indications for treatment developed by the Early Treatment of Retinopathy (ETROP) study group [1]. Despite timely treatment unfavourable outcomes can still occur. Unfavourable structural outcomes include macular fold, retinal detachment involving the macula or a retrolental mass obscuring the view of the posterior pole, and may occur in $9.1 \%$; unfavourable visual outcomes $(<1.85$ cycles on Teller acuity cards) may occur in $14.5 \%$ of infants [1]. In addition, the incidence of refractive errors, particularly myopia and astigmatism, is higher in infants who received laser photocoagulation for ROP than in children who did not require ROP treatment [2-5]. 
Table 1 Retreatment rates by ROP severity at presentation (reporting unit: right eyes)

\begin{tabular}{|c|c|c|c|c|c|c|c|c|c|}
\hline & Total $n$ & $\begin{array}{l}\text { At least one } \\
\text { retreatment }\end{array}$ & $\%$ & $\begin{array}{l}\text { Primary } \\
\text { treatment laser }\end{array}$ & $\begin{array}{l}\text { At least one } \\
\text { retreatment }\end{array}$ & $\%$ & $\begin{array}{l}\text { Primary treatment anti- } \\
\text { VEGF antibody }\end{array}$ & $\begin{array}{l}\text { At least one } \\
\text { retreatment }\end{array}$ & $\%$ \\
\hline APROP & 10 & 4 & 40 & 8 & 3 & 37.5 & 2 & 1 & 50 \\
\hline Type 1 & 107 & 13 & 12.1 & 96 & 9 & 9.4 & 11 & 4 & 36.4 \\
\hline $\begin{array}{l}\text { Type } 2 \\
\text { plus }\end{array}$ & 40 & 2 & 5 & 39 & 2 & 5.1 & 1 & 0 & \\
\hline Type 2 & 6 & 1 & 16.7 & 6 & 1 & 16.7 & 0 & 0 & \\
\hline Mild $^{\mathrm{a}}$ & $3^{\mathrm{a}}$ & 0 & 0.0 & 2 & 0 & 0.0 & 0 & 0 & \\
\hline \multirow[t]{2}{*}{ Partial RD } & 2 & 2 & 100 & 2 & 2 & 100 & 0 & 0 & \\
\hline & $168^{\mathrm{a}}$ & 22 & 13.1 & 153 & 17 & 11.1 & 14 & 5 & 35.7 \\
\hline
\end{tabular}

${ }^{\mathrm{a}} \mathrm{No}$ information on primary treatment was available for one infant.

Intravitreal injection of anti-vascular endothelial growth factor (VEGF) antibodies is an alternative to laser treatment in severe cases of ROP, such as aggressive posterior ROP (APROP), and ROP in zone 1 or posterior zone 2 [6]. Different from laser treatment, anti-VEGF antibody injection may allow maturation of the peripheral retina and anterior segment, and reports indicate better structural and visual outcomes (median VA of $0.3 \log$ MAR at around 2 years after treatment for type 1 ROP [7]) and a reduced incidence of myopia compared with laser $[6,8-16]$, though not all studies support these findings $[17,18]$. Anti-VEGF antibody therapy may require more than one treatment application, with reported retreatment rates ranging from 4 to $14 \%[6,9,11,12]$, though higher rates of $27 \%$ have also been reported [7]. Retreatment after laser is generally expected in around $13.9 \%$ [1], though recently higher retreatment rates of 26 to $32 \%$ have been reported $[6,19]$.

The aim of the present study was to report retreatment rates, visual and refractive outcomes in the national cohort of children treated for ROP over a 12-month period in the United Kingdom for whom we previously reported initial treatment data [20].

\section{Methods}

Our case ascertainment and data collection method have previously been described in detail [20]. In brief, between 01/12/2013 and 30/11/2014, ROP practitioners throughout the United Kingdom prospectively reported new cases of infants requiring treatment for ROP to the British Ophthalmic Surveillance Unit using an established reporting system. In addition, we set up an electronic UK ROPSpecial Interest Group. We sent practitioners who had reported ROP-treated infants' two case report forms: one after initial notification, and another 12 months later. Here we report data from the 12-month follow-up.

\section{Statistical analysis}

Data from the case report forms were entered onto an electronic Microsoft Office Access database. A random sample of forms was inspected to ensure data quality. Data were transferred into Stata version 14.0 for analysis. Characteristics of infants requiring ROP treatment were summarized using means and standard deviations for approximately Gaussian variables and medians and interquartile ranges (IQRs) for non-Gaussian continuous variables. Categorical variables are reported as numbers and proportions. This study was not powered to test for statistical significance of any prior hypotheses.

\section{Results}

\section{Children's characteristics}

We received 1-year follow-up forms for 168 children of the original cohort of 327 (51.4\%). Gestational age, age, birth weight, gender, ROP severity, primary treatment modality and number of treatments were similar in infants for whom follow-up data were available and those for whom followup data were not available.

At last follow-up, median age (IQR) was 14 (12-17.8) months. Seventy-six children $(45.2 \%)$ were girls. At the time of the follow-up report, 130 children $(77.4 \%)$ were under follow-up at the same unit where they had received ROP treatment. Fifteen children (8.9\%) were under the care of a different eye unit, 7 (4.2\%) were reported to have been discharged, 3 (1.8\%) lost to follow-up and 13 (7.74\%) had died. Causes of death were reported in six children, and included Beckwith-Wiedemann syndrome $(n=1)$, renal failure after laparotomy for necrotizing enterocolitis (1), severe bronchopulmonary dysplasia in extreme prematurity (1), severe hydrocephalus and respiratory failure (1), unknown (2). 
Table 2 Details of secondary, tertiary and any further treatment following initial laser or anti-VEGF antibody treatment, based on 168 children for whom one-year follow-up data were available (reporting unit: right eye)

\begin{tabular}{|c|c|c|c|c|}
\hline & \multicolumn{4}{|c|}{ Secondary treatment } \\
\hline & Argon laser & Anti-VEGF antibody & Missing data & Total \\
\hline \multicolumn{5}{|l|}{ Initial treatment } \\
\hline Argon laser & 5 & 7 & 2 & 14 \\
\hline VEGF inhibitor & 0 & 2 & 1 & 3 \\
\hline \multirow[t]{3}{*}{ Total } & 5 & 9 & 3 & 17 \\
\hline & \multicolumn{4}{|c|}{ Tertiary treatment } \\
\hline & Argon laser & Anti-VEGF antibody/plus laser & Surgery & Total \\
\hline \multicolumn{5}{|l|}{ Initial treatment } \\
\hline Argon laser & 3 & 0 & 2 & 5 \\
\hline VEGF inhibitor & 0 & 1 & 0 & 1 \\
\hline \multirow[t]{3}{*}{ Total } & 3 & 1 & 2 & 6 \\
\hline & \multicolumn{4}{|c|}{ Any further treatment at 1 year } \\
\hline & Argon laser & Anti-VEGF antibody/plus laser & Surgery & Total \\
\hline \multicolumn{5}{|l|}{ Initial treatment } \\
\hline Argon laser & 3 & 1 & 1 & 5 \\
\hline VEGF inhibitor & 0 & 2 & 0 & 2 \\
\hline Total & 3 & 3 & 1 & 7 \\
\hline
\end{tabular}

\section{Retreatment rates}

We calculated retreatment rates with a denominator of $n=$ 168 , the number of children for whom follow-up data were available (Table 1). Twenty-two of 168 (13.1\%) infants had at least one retreatment. Treatment was similar between the right and left eye; we report here the treatment details for the right eye (RE). Five of 14 REs which had anti-VEGF antibody injection as primary treatment (11 bevacizumab, 3 ranibizumab) required retreatment (35.7\%). Anti-VEGF antibody had been administered exclusively for APROP and type 1 ROP only; retreatment rates were 50 and $36.4 \%$, respectively. Retreatment rates after diode laser for APROP and type 1 ROP were 37.5 and 9.4\%, respectively (Table 1).

The retreatment given was laser in 8 REs, and antiVEGF antibody in 8 eyes, anti-VEGF plus laser for 2 eyes, surgery for 1 eye and laser plus surgery for 1 eye; retreatment information was not available for 2 eyes. Table 2 summarizes retreatment details.

The median interval from primary to secondary treatment was 17 (IQR 7-33, range 2-106) days, and the median interval from secondary to tertiary treatment was 36 (IQR 27-48, range 13-119) days.

For some infants, information about retreatment was available from the original incidence case report forms. We carried out a secondary analysis of retreatment rates, using all available retreatment information, with $n=327$, the original cohort population size, as denominator. Forty-two children $(12.8 \%)$ of the original cohort required at least one retreatment, so the results of this analysis were similar to our initial retreatment analysis (Supplementary material, Tables 5 and 6).

\section{Visual and refractive outcomes 1 year after ROP treatment}

Best-corrected visual acuity in $\log$ MAR in the RE was available in 46 children, and with both eyes open, 89 children. Median (IQR) RE acuity was $0.6(0.4-1.0) \log$ MAR, and median acuity with both eyes open was 0.4 (0.3-0.7) logMAR (Table 3). The median (IQR) spherical equivalent, calculated as spherical correction plus half of astigmatic correction, of REs was 0.44 ( -1.3 to 1.3 ) dioptre (D) $(n=116)$. Median (IQR) astigmatism of REs was 0.5 $(0-1.0) \mathrm{D}(n=111)$. Median (IQR) difference in refractive error between the two eyes was $0.125(0-0.75) \mathrm{D}(n=116)$. Twenty-four children $(20.5 \%)$ had been prescribed glasses.

The proportion of eyes with myopia of 5 dioptre sphere (DS) or more was highest in those with type 1 ROP: $5.3 \%$ (7 of 133 eyes) after laser, and 31\% (5 of 16 eyes) after anti-VEGF antibody. Overall, the proportion of eyes with myopia of $5 \mathrm{DS}$ or more was greater in the anti-VEGF antibody group than the laser group ( 26.3 vs. $6.7 \%, 5 / 19$ vs. 14/209 eyes) (Table 4). 


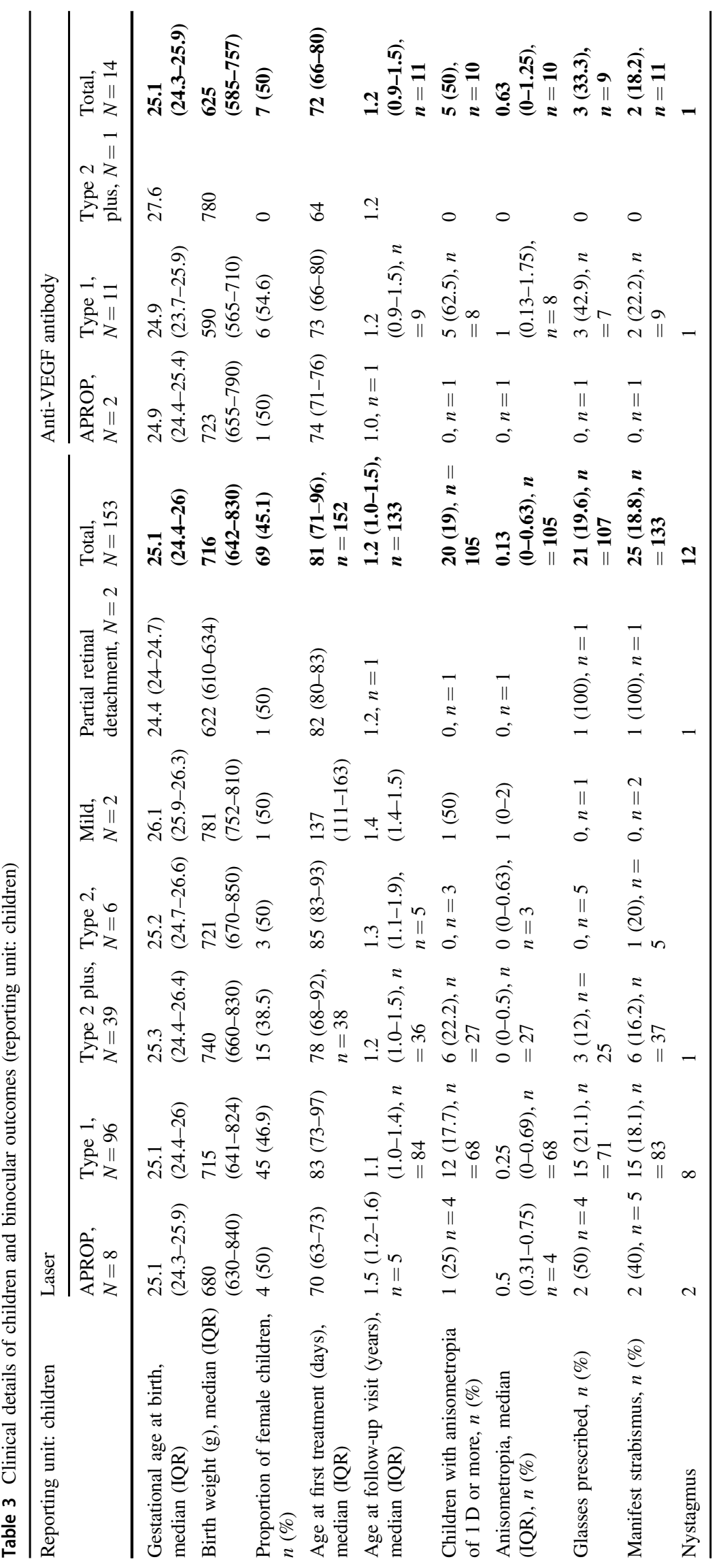




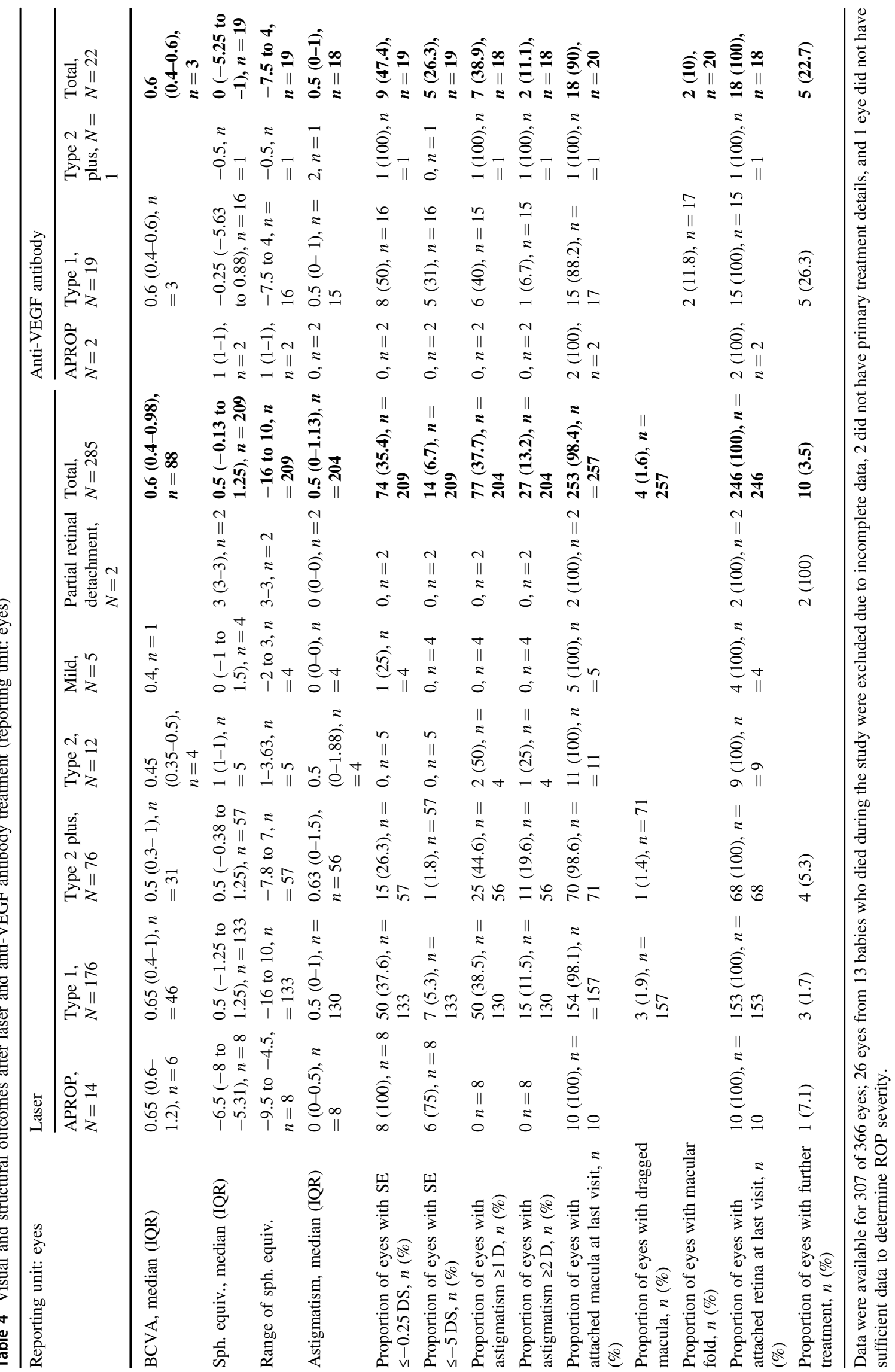


Eligibility for sight impairment certification was reported for 131 children. Eleven (8.4\%) were eligible to be certified as sight impaired, and five $(3.82 \%)$ as severely sight impaired $(3.8 \%)$.

\section{Anatomical outcomes 1 year after ROP treatment}

Unfavourable anatomical outcomes were reported in a total of six eyes (three each right and left eyes). Macular status at last follow-up was reported in 142 children. The macula of two REs (1.4\%) was 'dragged', and one RE $(0.7 \%)$ had a retinal fold involving the macula. Figures for left eyes were identical. The retina was attached in both eyes at the last follow-up visit in all children.

\section{Strabismus and nystagmus}

Twenty-one of 168 (12.5\%) children developed esotropia, 3 exotropia (1.8\%); none had a vertical deviation. Thirteen children $(7.7 \%)$ developed nystagmus.

\section{Other unfavourable ocular and neurological outcomes}

One child developed cataract $(0.6 \%)$, one glaucoma and one unilateral phthisis. Other structural ocular conditions were reported in three cases: residual vitreous haemorrhage (eye not given), optic atrophy and a previous exudative retinal detachment in one left eye. Amblyopia was reported in one child. Seven children (4.2\%) were diagnosed with cerebral visual impairment. Twenty children had neurological impairments: cerebral palsy in 7 (4.2\%), quadriplegia in 1, and developmental issues or motor delay in 8 children (4.8\%). Three children had hydrocephalus and 1 had periventricular haemorrhage. In addition, eight had a hearing impairment $(4.8 \%)$.

\section{Discussion}

The key findings of this study are that retreatment rates appear higher after initial anti-VEGF antibody than after laser, and that visual outcomes are generally good, with a median acuity with both eyes open of $0.4 \log$ MAR at age 14 months, which is within the normal range for this age when measured with acuity cards [21-23]. However, over $12 \%$ of children were eligible to be certified as sight impaired or severely sight impaired, comparable to the rate of unfavourable visual outcomes reported in the ETROP trial [1]. In addition, a high proportion of children $(20.5 \%)$ had started to wear glasses. Twenty children (12\%) had been diagnosed with conditions affecting the central nervous system.
The robust case ascertainment methodology which allowed collection of data at the national level is a strength of this study. A limitation is the high loss to follow-up since treatment, due to anonymized data collection and discharge from treating centres to referring units. Clinical assessment of visual acuity at the young age of a median of 14 months is often difficult, but the median we observed is within the normal range for this age. Reporting visual outcome might be more reliable at later ages, but further loss to follow-up may weaken data. Similarly, we report early refractive outcomes, 12 months after treatment, but myopia may develop or progress later. However, we observe a clear trend towards emmetropia and towards a greater prevalence of myopia even at this young age. Our study contributes to the ongoing discussion about advantages and disadvantages of anti-VEGF antibody treatment compared with diode laser. The retreatment rates we observe are in line with previous reports $[1,6,7]$. Whether retreatment rates after anti-VEGF antibody are truly higher than after diode laser will require further studies, ideally randomized controlled trials; one such trial is currently enroling participants (NCT02375971). Due to the high loss to follow-up and the possible selection bias towards inclusion of more severe cases, our figures may overestimate the actual retreatment rates.

As the majority of children in our study received laser treatment, we expected refractive outcomes similar to those reported by the ETROP trial: myopia of $-0.25 \mathrm{D}$ or greater in two-thirds of children, and of $5 \mathrm{D}$ or more in a quarter of children [24]. However, we found myopia of $-0.25 \mathrm{D}$ or more in only $36.4 \%$ (83/228 eyes), and high myopia of $5 \mathrm{D}$ or more in $8.33 \%$ (19/228 eyes), possibly due to the inclusion of children treated for milder forms of ROP in our study (type 2 plus, disease milder than type 1 with plus or pre-plus, e.g., zone 3 disease with plus or zone 2 stage 1 with plus) [20]. Median spherical equivalent was 0.44 DS, which is a favourable outcome for children who required ROP treatment, although it is lower than in healthy children and in children born prematurely, but without ROP [25]. Our observed rate of astigmatism of $1 \mathrm{D}$ or more $(37.8 \%$, $84 / 222$ eyes) is in line with the $32-42 \%$ reported by the ETROP trial and rates reported by other studies [2, 3]. Our prevalence of anisometropia of $1 \mathrm{D}$ or more $(21.7 \%, 25 / 115$ children) is higher than the $6.5 \%$ reported by others [3].

Twelve percent of children treated for ROP had been diagnosed with neurological or developmental problems by the age of 12-14 months, which is comparable with figures reported by the EPICURE studies [26]. As developmental concerns may become more apparent with increasing age, this figure may be an underestimate of long-term neurological impairments.

In conclusion, visual outcomes after treatment for ROP are good, with most children developing acuity normal for 
their age. Retreatment rates after initial anti-VEGF antibody appear higher than those after diode laser. Over $12 \%$ of infants have poor outcomes and are certified as sight impaired or severely sight impaired. Neurological impairments are common and affect $12 \%$ of children treated for ROP.

\section{Summary}

\section{What was known before}

- ROP is a sight-threatening condition with increasing incidence. New treatment in the form of anti-VEGF antibodies has entered clinical practice, with variable outcomes, possibly a higher need for long-term followup and re-treatments for recurrence of disease activity. Standard treatment remains diode laser photocoagulation of the non-vascularized retina, with increased risk of early emmetropization and myopia in childhood.

\section{What this study adds}

- Visual outcomes after treatment for ROP are good in the majority of children, though a significant minority have poor outcomes and are eligible for sight impairment certification. Retreatment rates appear higher after initial anti-VEGF antibody than after initial diode laser treatment. Refractive outcomes show a trend towards early emmetropia and myopia following diode laser, particularly in more severe ROP.

\footnotetext{
Acknowledgements We thank the British Ophthalmic Surveillance Unit, in particular Mr Barny Foot, for advice on study design and collection of case notifications. We also thank Miss Anneka Tailor for liaising with practitioners across the UK to facilitate data collection and completion of reports, and for maintaining the study database. We thank Mr Zabed Ahmed for setting up a comprehensive electronic database, and the Moorfields Special Trustees (grant ST $1401 \mathrm{D}$ ) and the Birmingham Eye Foundation for their generous funding for this work. The research was supported by the National Institute for Health Research (NIHR) Biomedical Research Centre based at Moorfields Eye Hospital NHS Foundation Trust and UCL Institute of Ophthalmology. The views expressed are those of the authors and not necessarily those of the NHS, the NIHR or the Department of Health. The study was approved by the Research Ethics Committee North of Scotland, Aberdeen (13/NS/0059). This work was registered at clinicaltrials.gov as NCT02484989.
}

Author contributions GGWA, CB, LB, AHD-N, VL and AR developed the study protocol. LB, AHD-N, GGWA and CB secured funding. All authors reviewed and discussed and interpreted the data acquired. WX carried out data analysis. AHD-N drafted the manuscript, which was then critically reviewed and modified by all authors.

Members of the UK ROP Special Interest Group: Abbott, Joseph; Aclimandos, Wagih; Adams, Gill; Al-Khaier, Ayman; Allen, Louise;
Arashvan, Kayvan; Ashworth, Jane; Barampouti, Faye; Barnes, Jonathan; Barrett, Victoria; Barry, John Sebastian; Bates, Adam; Berk, Tulin; Biswas, Susmito; Blaikie, Andrew; Brennan, Rosie; Bunting, Howard; Butcher, Jeremy; Butler, Lucilla; Chan-Ling, Tailoi; Chan, Jonathan; Child, Christopher; Choi, Jessy; Clark, David; Clifford, Luke; Dabbagh, Ahmad; Dahlmann-Noor, Annegret; Dawidek, Gervase; Dhir, Luna; Drake, Karen; Edwards, Richard; Esakowitz, Leonard; Escardo-Paton, Julia; Evans, Anthony; Fleck, Brian; Geh, Vernon; George, Nick; Gnanaray, Lawrence; Goyal, Raina; Haigh, Paul; Hancox, Joanne; Haynes, Richard; Heath, Dominic; Henderson, Robert; Hillier, Roxane; Hingorani, Melanie; Jain, Saurabh; Jain, Sunila; Jones, David; Kafil-Hussain, Namir; Kelly, Simon; Kenawy, Nihal; Kipioti, Tina; Kulkarni, Archana; Lavy, Tim; Laws, David; Lawson, Joanna; Leitch, Jane; Ling, Roland; Long, Vernon; Macrae, Mary; Mahmood, Usman; Markham, Richard; Marr, Jane; May, Kristina; McLoone, Eibhlin; Moosa, Murad; Morton, Claire; Mount, Ali; Muen, Wisam; Mulvihill, Alan; Munshi, Vineeta; Muqit, Mahi; Murray, Robert; Nair, Ranjit; Newman, William; O'Colmain, Una; Patel, Chetan; Patel, Himanshu; Pedraza, Luis Amaya; Pilling, Rachel; Puvanachandra, Narman;Quinn, Anthony; Rathod, Dinesh; Reddy, Aravind; Reddy, Ashwin; Rowlands, Alison; Scotcher, Stephen; Scott, Christopher; Sekhri, Rajnish; Shafiq, Ayad; Sleep, Tamsin; Tambe, Katya; Tandon, Anamika; Tappin, Alison; Taylor, Robert; Theodoro, Maria; Thomas, Shery; Thompson, Graham; Tiffin, Peter; Ullah, Muhammed Aman; Watts, Patrick; West, Stephanie; Whyte, Iain; Wickham, Louisa; Williams, Cathy; Wong, Chien; Wren, Siobhan; Zakir, Rahila.

\section{Compliance with ethical standards}

Conflict of interest This work was funded by Moorfields Eye Charity and the Birmingham Eye Foundation. There have not been any financial relationships with any organizations that might have an interest in the submitted work in the previous 3 years and no other relationships or activities that could appear to have influenced the submitted work. The funders have not in any way influenced the study design or conduct, and the researchers are independent from the funders. All authors, external and internal, had full access to all of the data (including statistical reports and tables) in the study and can take responsibility for the integrity of the data and the accuracy of the data analysis. The lead author, AHDN, affirms that the manuscript is an honest, accurate, and transparent account of the study being reported; that no important aspects of the study have been omitted; and that any discrepancies from the study as planned have been explained.

\section{References}

1. Early Treatment For Retinopathy Of Prematurity Cooperative G. Revised indications for the treatment of retinopathy of prematurity: results of the early treatment for retinopathy of prematurity randomized trial. Arch Ophthalmol. 2003;121:1684-94.

2. Davitt BV, Dobson V, Quinn GE, Hardy RJ, Tung B, Good WV, et al. Astigmatism in the Early treatment for retinopathy of prematurity study: findings to 3 years of age. Ophthalmology. 2009;116:332-9.

3. Holmstrom M, el Azazi M, Kugelberg U. Ophthalmological longterm follow up of preterm infants: a population based, prospective study of the refraction and its development. $\mathrm{Br} \mathrm{J}$ Ophthalmol. 1998;82:1265-71.

4. Saunders KJ, McCulloch DL, Shepherd AJ, Wilkinson AG. Emmetropisation following preterm birth. $\mathrm{Br} \mathrm{J}$ Ophthalmol. 2002;86:1035-40.

5. Deng L, Gwiazda JE. Anisometropia in children from infancy to 15 years. Invest Ophthalmol Vis Sci. 2012;53:3782-7. 
6. Mintz-Hittner HA, Kennedy KA, Chuang AZ. Group B-RC Efficacy of intravitreal bevacizumab for stage 3+ retinopathy of prematurity. N Eng J Med. 2011;364:603-15.

7. Chuluunbat T, Chan RV, Wang NK, Lien R, Chen YP, Chao AN, et al. Nonresponse and recurrence of retinopathy of prematurity after intravitreal ranibizumab treatment. Ophthalmic Surg Lasers Imaging Retina. 2016;47:1095-105.

8. Geloneck MM, Chuang AZ, Clark WL, Hunt MG, Norman AA, Packwood EA, et al. Refractive outcomes following bevacizumab monotherapy compared with conventional laser treatment: a randomized clinical trial. JAMA Ophthalmol. 2014;132:1327-33.

9. Mintz-Hittner HA, Geloneck MM, Chuang AZ. Clinical managemen of recurrent retinopathy of prematurity after intravitreal bevacizumab monotherapy. Ophthalmology. 2016;123:1845-55.

10. Yoon JM, Shin DH, Kim SJ, Ham DI, Kang SW, Chang YS, et al. Outcomes after laser versus combined laser and bevacizumab treatment for type 1 retinopathy of prematurity in zone I. Retina. 2017;37:88-96.

11. Gunay M, Sukgen EA, Celik G, Kocluk Y. Comparison of bevacizumab, ranibizumab, and laser photocoagulation in the treatment of retinopathy of prematurity in Turkey. Curr Eye Res. 2016;42:1-8.

12. Hwang CK, Hubbard GB, Hutchinson AK, Lambert SR. Outcomes after intravitreal bevacizumab versus laser photocoagulation for retinopathy of prematurity: a 5-year retrospective analysis. Ophthalmology. 2015;122:1008-15.

13. Harder BC, von Baltz S, Jonas JB, Schlichtenbrede FC. Intravitreal low-dosage bevacizumab for retinopathy of prematurity. Acta Ophthalmol (Copenh). 2014;92:577-81.

14. O'Keeffe N, Murphy J, O'Keefe M, Lanigan B. Bevacizumab compared with diode laser in stage 3 posterior retinopathy of prematurity: a 5 year follow up. Ir Med J. 2016;109:355.

15. Sankar MJ, Sankar J, Mehta M, Bhat V, Srinivasan R. Antivascular endothelial growth factor (VEGF) drugs for treatment of retinopathy of prematurity. Cochrane Database Syst Rev. 2016;2: CD009734.

16. Gunay M, Celik G, Gunay BO, Aktas A, Karatekin G, Ovali F. Evaluation of 2-year outcomes following intravitreal bevacizumab (IVB) for aggressive posterior retinopathy of prematurity. Arq Bras Oftalmol. 2015;78:300-4.
17. Kabatas EU, Kurtul BE, Altiaylik Ozer P, Kabatas N. Comparison of intravitreal bevacizumab, intravitreal ranibizumab and laser photocoagulation for treatment of type 1 retinopathy of prematurity in Turkish preterm children. Curr Eye Res. 2017;42: 1054-1058.

18. Gunay M, Sekeroglu MA, Bardak H, Celik G, Esenulku CM, Hekimoglu E, et al. Evaluation of refractive errors and ocular biometric outcomes after intravitreal bevacizumab for retinopathy of prematurity. Strabismus. 2016;24:84-88.

19. Holmstrom G, Hellstrom A, Jakobsson P, Lundgren P, Tornqvist K, Wallin A. Five years of treatment for retinopathy of prematurity in Sweden: results from SWEDROP, a national quality register. Br J Ophthalmol. 2016;100:1656-61.

20. Adams GG, Bunce C, Xing W, Butler L, Long V, Reddy A, et al. Treatment trends for retinopathy of prematurity in the UK: active surveillance study of infants at risk. BMJ Open. 2017;7: e013366.

21. Adoh TO, Woodhouse JM. The Cardiff acuity test used for measuring visual acuity development in toddlers. Vision Res. 1994;34:555-60.

22. Courage ML, Adams RJ. Visual acuity assessment from birth to three years using the acuity card procedure: crosssectional and longitudinal samples. Optom Vis Sci. 1990;67: 713-8.

23. Sharma P, Bairagi D, Sachdeva MM, Kaur K, Khokhar S, Saxena R. Comparative evaluation of Teller and Cardiff acuity tests in normals and unilateral amblyopes in under-two-year-olds. Indian $\mathrm{J}$ Ophthalmol. 2003;51:341-5.

24. Davitt BV, Dobson V, Good WV, Hardy RJ, Quinn GE, Siatkowski RM, et al. Prevalence of myopia at 9 months in infants with high-risk prethreshold retinopathy of prematurity. Ophthalmology. 2005;112:1564-8.

25. Quinn GE, Dobson V, Davitt BV, Hardy RJ, Tung B, Pedroza C, et al. Progression of myopia and high myopia in the early treatment for retinopathy of prematurity study: findings to 3 years of age. Ophthalmology. 2008;115:1058-64 e1051.

26. Moore T, Hennessy EM, Myles J, Johnson SJ, Draper ES, Costeloe KL, et al. Neurological and developmental outcome in extremely preterm children born in England in 1995 and 2006: the EPICure studies. BMJ. 2012;345:e7961. 